Supporting Information

\title{
Controlled Silanization: High Molecular Regularity of Functional Thiol Groups on Siloxane Coatings
}

\author{
Tien-Jung Lee, ${ }^{\#}$ Lai-Kwan Chau, ${ }^{\S}$ Chun-Jen Huang ${ }^{\dagger}, \#, \| * *$ \\ ${ }^{\dagger}$ Department of Biomedical Sciences and Engineering, ${ }^{\#}$ Department of Chemical and \\ Materials Engineering, National Central University, Jhong-Li, Taoyuan 320, Taiwan
}

॥R\&D Center for Membrane Technology, Chung Yuan Christian University, 200

Chung Pei Rd., Chung-Li City 32023, Taiwan

${ }^{\S}$ Department of Chemistry and Biochemistry and Center for Nano Bio-Detection,

National Chung Cheng University, Chiayi 621, Taiwan

*Corresponding author: cjhuang@ncu.edu.tw (C.-J.H.) 

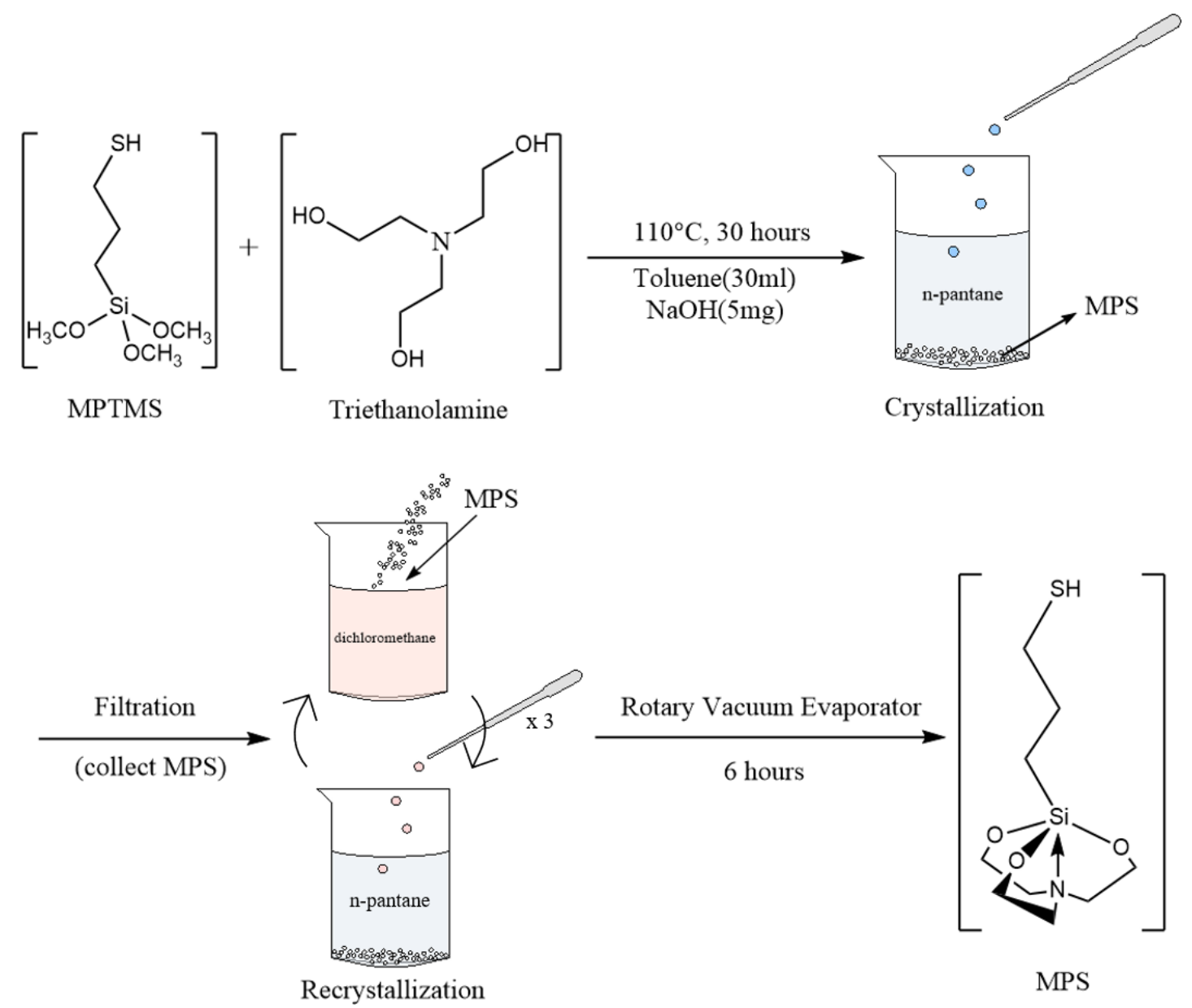

Scheme S1. Overall workflow of the MPS synthesis.

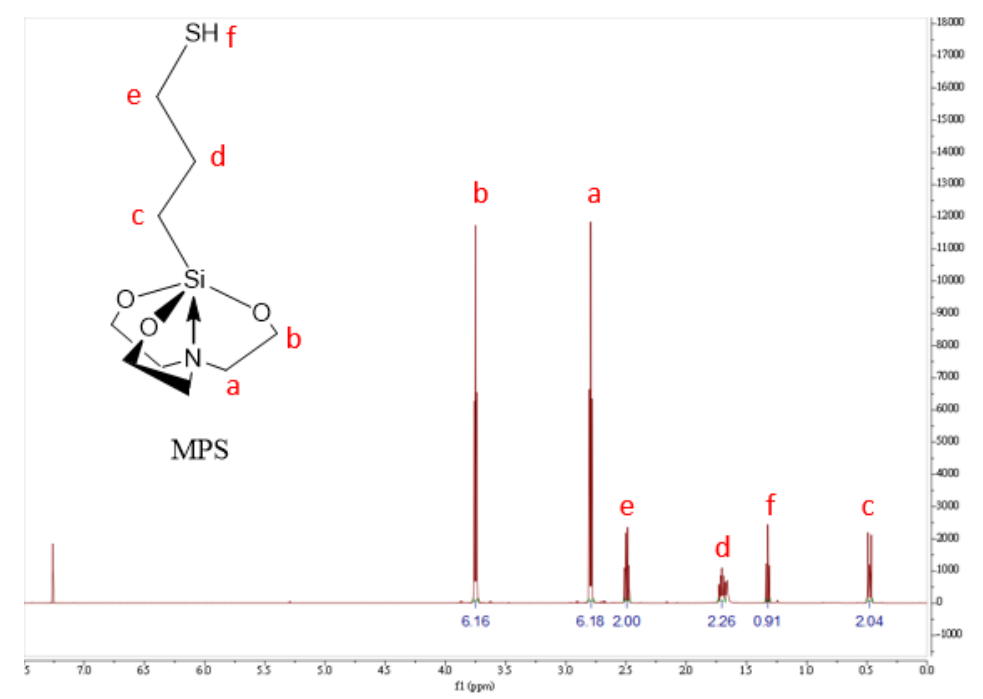

Figure S1. NMR spectra for MPS. 